\title{
An improved foolproof device for eggs' sterilization of Corcyra cephalonica (Stainton)
}

\author{
Surender Kumar Singh* and P. Kumar \\ National Research Centre for Integrated Pest Management, IARI, Pusa Campus, New Delhi-110012, INDIA \\ *Corresponding author. E-mail: sk_ncipm@yahoo.com \\ Received: August 8, 2016; Revised received: February 19, 2017; Accepted: June 28, 2017
}

\begin{abstract}
Mass rearing of numerous biological control agents depends on large amounts of eggs of factitious hosts like rice meal moth, Corcyra cephalonica (Stainton) (Lepidoptera : Pyralidae). Corcyra eggs sterilization is required before they are used for parasitisation by Trichogrammatids. To meet the requirement of eggs sterilization of this insect, a new innovative device was invented, fabricated and studied. It consisted of an enclosed structure having semi-circular shaped drawer. An ultra violet (UV) tube of $30 \mathrm{~W}$ is fixed on the centre of its ceiling to maintain a uniform distance of $35 \mathrm{~cm}$ from the eggs to be sterilized. An exposure of 10 minutes to eggs of C. cephalonica was required to get optimum sterilization. As whole body of the device was an enclosed structure, hence, there was no exposure of UV rays to the laboratory workers. The different sized-models $(150,100,50$ of "egg cards" required for Trichogrammatids) can be fabricated to meet the diverse requirements. Irradiated eggs were found having no any adverse effects on the abilities of Trichogrammatids parasitisation and its emergence. It can also used for UV sterilization of laboratory materials like glassware, plastic ware, clothes, cotton etc. The period of exposure to the UV light was set using timer. This innovative UV chamber had numerous advantages i.e., the UV rays used had damaged the host embryo. The damaged embryo could not develop into next stage, at the same time damaged embryo found suitable for parasitization of Trichogrammatids. The whole body of the device was made-up of an enclosed structure; hence, there was no exposure of UV rays to the laboratory workers. The different sized-models $(150,100,50$ of "egg cards" required for Trichogrammatids) can be fabricated to meet the diverse requirements of clients. As the UV chamber was made up of metal and ply boards, hence, it was durable. The longitudinal flanges provided in the drawer of the device prevented the host egg bearing cards to glide one over other from curved surface. The device was found safe and effective for sterilization of Corcyra eggs and easy to operate.
\end{abstract}

Keywords: Corcyra cephalonica, Egg sterilization, Mass rearing of Trichogrammatids, UV sterilization chamber

\section{INTRODUCTION}

Biological control approach of crop pests offers a viable, sustainable, ecological and economical solution to the pest problems. Rice meal moth, Corcyra cephalonica (Stainton) (Lepidoptera :Pyralidae) a stored grain insect pest is industrialized for mass production of many predators and parasitoids in the laboratory. Among biological control agents, Trichogrammatid parasitoids were used worldwide (Senthil-Nathan et al., 2006), as they were found attacking the eggs of over 200 insect species (Mansfield and Mills, 2004). Mass rearing of Trichogramma spp. is an economically feasible methodology for control of many Lepidopteran pests (Wang et al., 2012). Trichogrammatid parasitize eggs of Lepidoptera by laying one or more eggs inside the eggs of the host insects. Tuncbilek et al. (2012) studied the potential improvements in the suitability of host eggs for mass-rearing of Trichogrammatid through pre-exposure of $C$. Cephalonica eggs to radiation. Irradiation plays significant role in the production of natural enemies. It has been reported that the parasitisation increased in the irradiated hosts (Hamed et al., 2009 and Tuncbilek et al., 2009) as irradiated eggs were found more suitable as compared to normal for parasitoid development. Hendrichs et al. (2009) reported that irradiated eggs can be used as supplemental host individuals without fearing that the released host insects themselves be converted into pests. The sterilization of $C$. Cephalonica eggs is required to avoid emergence of its larvae as these eggs are used for mass-multiplication of Trichogrammatid egg parasitoids. As there was no proper device for $C$. Cephalonica eggs sterilization, therefore, the present study was done and an innovative device was invented, up-scaled and standardized.

\section{MATERIALS AND METHODS}

For mass production of Trichogrammatid, Corcyra eggs need to be sterilized before use and for quick and uniform sterilization of the eggs, a new type of sterilization chamber was conceptualized, designed, fabricated, up-scaled and standardized (Fig.1). It was made-up of metal and ply boards. The chamber comprised a 


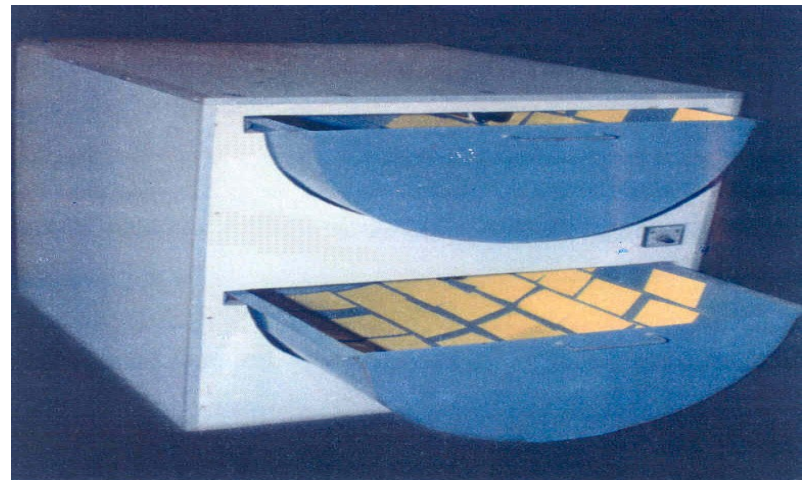

Fig. 1. An improved fool-proof device (105-cm long, $88-\mathrm{cm}$ broad and 105-cm high) for eggs' sterilization of Corcyra cephalonica (Stainton).

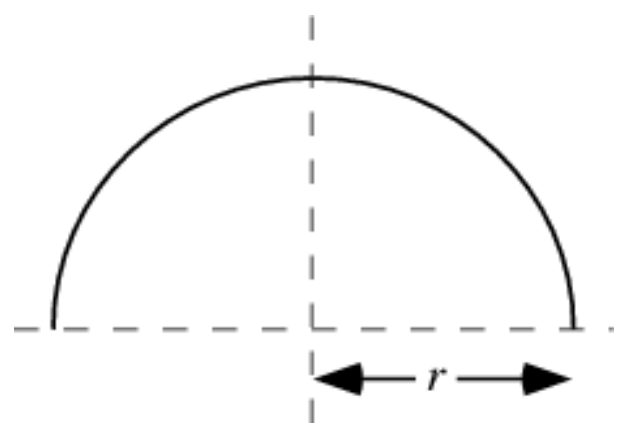

Fig. 2. Semicircle drawer having uniform radius in the newly conceptualized UV device.

cuboid box, $105-\mathrm{cm}$ long, $88-\mathrm{cm}$ broad and $105-\mathrm{cm}$ high. It was closed on all sides except from the front and was divided into two equal parts by a horizontal partition. Each partition was provided with a drawer which had semi-circular base of $35 \mathrm{~cm}$ radius. The cross section of this drawer was semi-circular. The length of the drawer was $100 \mathrm{~cm}$. Inside the semicircular chamber, the drawer was provided with longitudinal flanges of 2-mm thickness and 9-cm apart. These flanges were provided to prevent egg bearing cards to glide one over other from curved surface. Postcard size cards (75 in total) bearing eggs (approx. 1 cubic $\mathrm{cm}$ eggs/card) can be kept in one drawer. In the outer surface-side, the drawer was provided with channels and wheels. With these provisions, the drawers can be pulled out and pushed back in place easily. The ceiling of each drawer was provided with a $30 \mathrm{~W}$ UV tube-light. Each tube was connected with a timer.

For the sterilization of Corcyra cephalonica eggs, the freshly laid eggs were glued on the post-card size cards (approx. $13.5 \mathrm{~cm}$ length $\mathrm{x} 9 \mathrm{~cm}$ width). Such cards are called as "egg card" or "Trichocards" as it being to be used for parasitisation by Trichogrammatid egg parasitoids. Precautions were taken to ensure that glue film remain thin enough and dry enough so that the eggs were not immersed in it but just remain stick by a small contact with the card. The eggs were uniformly sprinkled on the egg cards on its upper surface and then these were arranged in the longitudinal flanges of the semi-circular drawer. The drawer was closed and the timer was set for a required period of time. The UV light source was switched on. The "egg cards" were exposed to ultra violet rays (30 W UV-tube) for 10 minutes in the enclosed chamber having a uniform distance of $35 \mathrm{~cm}$ from the UV tube.

To further test the performance of the device, a total of two treatments (treatment-1 UV exposure to egg cards and Treatment-2/control - no UV exposure to egg cards) were done. In treatment-1, a total of 18 egg cards were put randomly inside the drawer of the device and these were exposed to UV rays (30 W UVtube) for a period of 10 minutes. After treatment, a set of two egg cards (total 3 repeats) were taken out and then these were kept in the polythene bags separately and the mouth of these bags were tightened with the help of elastic bands and these were kept for a period of ten days in the laboratory to observe the emergence of $C$. Cephalonica larvae, if any. Another set of two egg cards (total 3 repeats) were offered for parasitisation to Trichogramma japonicum Ashmead and T. chilonis Ishii each to find out the parasitisation and adult emergence. Such emerged Trichogrammatid from treated egg cards were further offered UV treated egg cards to find out the parasitism and adult emergence subsequently. Simultaneously, a total of six untreated egg cards were kept separately as control and emergence of larvae of C. Cephalonica were observed from these egg cards.

\section{RESULTS AND DISCUSSION}

An innovative prototype of the newly conceptualized UV device was fabricated and standardized. When the egg-cards were exposed to the UV radiation in the device, it was found that there was $100 \%$ mortality of the embryos of the eggs of C. cephalonica as there was no emergence of larvae from these UV treated eggcards. On the other hand, the emergences of larvae of C. Cephalonica from untreated egg-cards were found. Due to the central position of UV light source in the ceiling of the device, it maintains equidistance $(35 \mathrm{~cm})$ from the bottom of the drawer and thus uniform radiation was put on the egg-cards and thus all the embryos of the eggs of $C$. Cephalonica were found dead in the treated egg-cards. It is well-established principle that a circle contains uniform radius (' $r$ ') and all points on the arc of a circle always remain equidistance from the centre of the circle (Fig. 2). Utilizing this fundamental basic principle of the circle, the drawer of the UV device was designed and fabricated in semi-circular shape and the effect of UV radiation was found uniform as there was $100 \%$ mortality of the embryos of the eggs. Similar effect of UV radiation on mortality of C. cephalonica eggs was found by Singh et al., 1994. It was found that the irradiated "egg cards" did not have any adverse effects on the abilities of $T$. japoni- 
cum and T. chilonis parasitisation. Successful parasitisation and subsequently normal adults' emergence was found from these irradiated eggs. They had normal body shape, structure and normal ability to further parasitize the egg-cards as offered subsequently. UV rays used had damaged the host embryo due to smaller size and thin outer layer of the eggs; the damaged embryo could not develop into next stage. At the same time, damaged embryo had helped the Trichogrammatid for successful parasitization. Faruki et al. (2007) also observed decrease in egg hatching and adult emergence in UV radiated eggs of Tribolium castaneum, T. confusum and the almond moth, Cadra cautella. Herlin et al. (2015) had also observed effect of UV radiation on the life cycle of rice moth Corcyra cephalonica (Stainton) (Lepidoptera: Pyralidae) and disruption in hatchability.

As the egg cards were exposed to UV radiations in a closed semi-circular chamber, hence undesired exposure to UV radiations was ruled out. This drawer can be easily pulled out of or pushed into this chamber as it is being provided with the channels and the wheels. There was no sliding of "egg cards" over one-another as the drawer was being provided with longitudinal flanges of 2-mm thickness. To meet the requirements of different laboratories, the up-scaled foolproof device for eggs sterilization of $C$. Cephalonica can be made of various capacities viz., for the sterilization of $150,100,50$ Trichocards or any other similar capacities by changing the dimensions of the drawer, channels, Trichocards etc. It can be easily made multistoried chambered. It can also used for the UV sterilization of laboratory materials like glassware, plastic ware, clothes, cotton etc. Since there are two drawers, the time required to arrange the egg cards in one drawer was sufficient to sterilize the cards in other drawer. Thus one can sterilize 75 cards per 10 minutes using these drawers alternately. As the device was made-up of metal and ply boards, hence, it was durable. The accessories like installations of handles and wheels for to and fro movements of the device in the laboratories etc. can be installed in it as per the requirements.

\section{Conclusion}

An innovative prototype of UV device for eggs' sterilization of $C$. cephalonica was fabricated and standardized. The drawer of the UV device was designed and fabricated in semi-circular shape (having uniform radius) for uniform UV radiation on egg cards. Due to uniform radiation on egg-cards, there was $100 \%$ mortality of the embryos of the eggs. The UV rays used had damaged the host embryo. The damaged embryo could not develop into next stage. At the same time, damaged embryo had helped the Trichogrammatid for successful parasitisation and emergence. These emerged Trichogrammatid had normal body shape and structure and had ability to further parasitize and emergence in subsequent generation. Advantages of this method were numerous i.e., the radiation helped the parasitoids to parasitize eggs without any difficulty and if the eggs escaped from the parasitoid, the radiation had already damaged the host eggs, therefore, there was no hatching of $C$. cephalonica eggs. As the egg cards were exposed to UV radiations in a closed semi-circular chamber, the undesired exposure to UV radiations was ruled out. The drawer was provided with channels and wheels and with these provisions, the drawers can be pulled out and pushed back in place easily.

\section{REFERENCES}

Faruki, S. I., Das, D. R., Khan, A. R. and Khatun, M. (2007). Effects of ultraviolet $(254 \mathrm{~nm})$ irradiation on egg hatching and adult emergence of the flour beetles, Tribolium castaneum, T. confusum and the almond moth, Cadra cautella. Journal of Insect Science, 7:1-6.

Hamed, M., Sajid, N. and Asia, R. (2009). Use of gamma radiation for improving the mass production of Trichogramma chilonis and Chrysoperla carnea. Bio Cont. Sci. and Tech., 19: 43-48.

Herlin, Sheeja C., Stevens Jones R. D., Sam Manohara Dhas, S. (2015). Effect of UV radiation on the life cycle of rice moth Corcyra cephalonica (Stainton) (Lepidoptera: Pyralidae). International Journal of Multidisciplinary Research and Development, 2(6): 153-154.

Hendrichs, J., Kenneth, B., Gernot, H., Carpenter, J. E., Greany, P. and Robinson, A. S. (2009). Improving the cost-effectiveness, trade and safety of biological control for agricultural insect pests using nuclear techniques. Bio Cont. Sci. and Tech., 19: 3-22.

Mansfield, S. and Mills, N. J. (2004). A comparison of methodologies for the assessment of host performance of the gregarious egg parasitoid Trichogramma platneri. Biol. Control, 29: 332-340.

Senthil-Nathan, S., Kalaivani, K., Mankin, R. W. and Murugan, K. (2006). Effects of millet, wheat, Rice, and sorghum diets on development of Corcyra cephalonica (Stainton) (Lepidoptera, Galleriidae) and its suitability as a host for Trichogramma chilonis Ishii (Hymenoptera, Trichogrammatidae). Environ. Entomol., 35, 784-788.

Singh, Sangeeta, Paul, A. V. N. and Deepali, Bharti (1994). Effect of UV radiation on mortality of Corcyra cephalonica, Stainton (Lepidoptera: Gelechiidae) eggs. Indian J. Ento., 56(4):347-351.

Tuncbilek, A. S., Ulku, C. and Fahriye, S. (2009). Suitability of irradiated and cold stored eggs of Ephestia kuehniella (Pyralidae: Lepidoptera) for stockpiling the eggs-parasitoid Trichogramma evanescens (Trichogrammatidae: Hymenoptera) in diapause. Bio Cont. Sci. and Tech., 19: 127138.

Tuncbilek, A.S., Ercan, F.S. and Canpolat, U. (2012). Effect of ionizing (gamma) and non-ionizing (UV) radiation on the development of Trichogramma euproctidis (Hymenoptera : Trichogrammatidae). Arch. Biol. Sci., 64:287-295.

Wang, Y., Yu, R., Zhao, X., Chen, L., Wu, C., Chang, T. and Wang, Q. (2012). Susceptibility of adult Trichogramma nubilale (Hymenoptera, Trichogrammatidae) to selected insecticides with different modes of action. Crop Prot., $3476-82$. 